\title{
Autorisations d'urbanisme, principe de précaution et responsabilité pénale de l'élu local en cas de catastrophe naturelle
}

Town-Planning Permission, the Principle of Precautionary Measures and the Criminal Liability of the Locally Elected Official in the Event of a Natural

Disaster

Dorothée Goetz

\section{(2) OpenEdition}

\section{Journals}

Édition électronique

URL : https://journals.openedition.org/crdf/583

DOI : $10.4000 /$ crdf.583

ISSN : 2264-1246

Éditeur

Presses universitaires de Caen

\section{Édition imprimée}

Date de publication : 1 novembre 2016

Pagination : $21-29$

ISBN : 978-2-84133-838-2

ISSN : $1634-8842$

Référence électronique

Dorothée Goetz, « Autorisations d'urbanisme, principe de précaution et responsabilité pénale de l'élu local en cas de catastrophe naturelle », Cahiers de la recherche sur les droits fondamentaux [En ligne], 14 | 2016, mis en ligne le 01 octobre 2019, consulté le 14 novembre 2022. URL : http:// journals.openedition.org/crdf/583; DOI : https://doi.org/10.4000/crdf.583 


\title{
Autorisations d'urbanisme, principe de précaution et responsabilité pénale de l'élu local en cas de catastrophe naturelle
}

\author{
Dorothée GOETZ \\ Docteure en droit privé et sciences criminelles de l'université de Strasbourg
}

I. Principe de précaution et responsabilité pénale: une rencontre en principe impossible

A. La logique de précaution déjà présente dans le droit pénal

B. L'obstacle infranchissable du lien de causalité

II. Principe de précaution et responsabilité pénale: une rencontre à empêcher

A. Une distinction à préserver entre le principe de précaution et l'imprudence

B. La préservation du rôle de la responsabilité pénale

Version moderne et dépoussiérée du principe de prudence considéré par Tocqueville comme inhérent à l'émergence de la démocratie moderne, le principe de précaution est devenu un élément incontournable et controversé de la pensée juridique moderne ${ }^{1}$ qui traduit une demande sociale et politique ${ }^{2}$. Son étude est inévitable pour ceux qui nourrissent un intérêt pour l'environnement et, plus généralement, pour les rapports entre la science et le droit. MM. Tixier et Elfassi définissent le principe de précaution comme:

[...] un principe d'action qui impose que l'autorité compétente prenne des mesures appropriées en face d'un risque non avéré, mais dont la potentialité n'a pas pu être écartée avec certitude par les connaissances scientifiques à une époque donnée ${ }^{3}$.

Dans leur rapport remis au Premier ministre en 1998, $\mathrm{M}$. Kourilsky et $\mathrm{M}^{\mathrm{me}}$ Viney invitent à penser le principe de précaution en tant que règle de conduite, voire en tant que standard juridique. Ils précisent en effet que:

Le principe de précaution définit l'attitude que doit observer toute personne qui prend une décision concernant une activité dont on peut raisonnablement supposer qu'elle comporte un danger grave pour la santé ou la sécurité des générations actuelles ou futures, ou pour l'environnement ${ }^{4}$.

1. Voir L. Benoit, «Le principe de précaution reste... un principe», Environnement, n 4, avril 2005, p. 27; Y. Jegouzo, «De certaines obligations environnementales: prévention, précaution et responsabilité», L'actualité juridique. Droit administratif, 2005, p. 1164-1169.

2. Voir D. Chagnollaud, "Le principe de précaution est-il soluble dans la loi ? À propos de l'article 5 de la charte de l'environnement», Recueil Dalloz, 2004, p. 1103; M. Franc, "Traitement juridique du risque et principe de précaution", L'actualité juridique. Droit administratif, 2003, p. 360 ; B. Pignerol, «Responsabilité et socialisation du risque», L'actualité juridique. Droit administratif, 2005, p. 2211; P. Kourilsky, G. Viney, Le principe de précaution: rapport au Premier ministre, Paris, O. Jacob - La documentation française, 2000.

3. J.-L. Tixier, P. Elfassi, «Le principe de précaution: un relais efficace du pouvoir de police en matière de téléphonie mobile?», Collectivités territoriales - intercommunalité, 2001, p. 4.

4. P. Kourilsky, G. Viney, Le principe de précaution..., p. 215. 
Si, pour d'aucuns, sa définition exacte serait introuvable dans les textes ${ }^{5}$, la présente contribution s'appuiera sur deux dispositions dotées d'une valeur juridique et qui permettent de dessiner les contours de la précaution. Premièrement, l'article $1^{\text {er }}$ de la loi no 95-101 du 2 février 1995, dite loi Barnier, codifié à l'article L. 110-1 du Code de l'environnement. Le principe de précaution y est défini comme:

Le principe $[\ldots]$ selon lequel l'absence de certitudes, compte tenu des connaissances scientifiques et techniques du moment, ne doit pas retarder l'adoption de mesures effectives et proportionnées visant à prévenir un risque de dommages graves et irréversibles à l'environnement à un coût économiquement acceptable.

Deuxièmement, l'article 5 de la Charte de l'environnement établie par la loi constitutionnelle $\mathrm{n}^{\circ}$ 2005-205 du $1^{\text {er }}$ mars 2005. Cette disposition précise que:

Lorsque la réalisation d'un dommage, bien qu'incertaine en l'état des connaissances scientifiques, pourrait affecter de manière grave et irréversible l'environnement, les autorités publiques veillent, par application du principe de précaution et dans leurs domaines d'attributions, à la mise en œuvre de procédures d'évaluation des risques et à l'adoption de mesures provisoires et proportionnées afin de parer à la réalisation du dommage.

Il ressort de ces dispositions que le principe de précaution repose sur deux composantes essentielles: l'existence d'un risque scientifiquement incertain et la nécessité d'agir malgré l'incertitude scientifique. Ainsi, il s'agit d'éviter que ne survienne un « risque virtuel ${ }^{6}$ que nous pourrions appeler le risque du risque. Plusieurs drames parmi lesquels l'affaire du sang contaminé ${ }^{7}$, la crise de la vache folle ${ }^{8}$ ou encore de l'amiante ${ }^{9}$ ont montré que n'intervenir que lorsque la connaissance scientifique du risque est établie peut s'avérer inopérant. C'est pourquoi la philosophie du principe de précaution est qu'il n'est plus nécessaire de savoir pour agir. Toutefois, il découle de l'utopie du « risque zéro » que son ambition ne saurait être l'élimination de tous les risques. Le pragmatisme juridique invite plutôt à concevoir la précaution comme une clé susceptible de limiter leur probabilité de réalisation. Ce faisant, en ouvrant la voie à une « superprévention $»^{10}$, la logique de précaution enrichit «tous les domaines [dont le droit pénal fait naturellement partie] où la sécurité collective est en cause ${ }^{11}$ d'une nouvelle dimension.

Entre ce principe, le respect des règles d'urbanisme ${ }^{12}$ et la responsabilité pénale d'un agent décisionnaire, la rencontre était inévitable. En effet, en enrichissant le concept d'intérêt général, le principe de précaution est étroitement lié aux prises de décision des agents publics lorsqu'ils délivrent des autorisations ou des refus d'autorisation en matière d'urbanisme. La situation qui va nous intéresser est celle dans laquelle se produit une catastrophe naturelle postérieurement à la délivrance d'une autorisation d'urbanisme (par exemple, un permis de construire). Dans sa thèse, $\mathrm{M}^{\mathrm{me}}$ Lacroix définit la catastrophe comme:

[...] un événement ponctuel ou sériel, d'origine naturelle ou et/ ou anthropique, susceptible de recevoir une qualification pénale, causant d'importants dégâts matériels et / ou de nombreuses victimes et générateur d'un fort impact émotionnel, qui, à ce titre, nécessite la mise en œuvre de mesures spécifiques dans l'intérêt des victimes ${ }^{13}$.

M. le professeur Lienhard évoque même la nécessité de créer un "droit des catastrophes ${ }^{14}$ consacré, et malheureusement la liste est non exhaustive, aux accidents industriels, aux accidents technologiques, aux accidents de transport routier, aérien, maritime, ferroviaire, aux effets de foule non maîtrisés, aux effondrements ou incendies d'immeubles, aux produits défectueux et aux catastrophes naturelles. L'article $1^{\text {er }}$ de la loi no $82-600$ du 13 juillet $1982^{15}$ relative à l'indemnisation des victimes de catastrophes naturelles indique que:

5. N. de Sadeleer, "Les avatars du principe de précaution en droit public: effet de mode ou révolution silencieuse?», Revue française de droit administratif, 2001, p. 547: «[...] le propre d'une telle norme est précisément de ne pas faire l'objet d'une définition complète dans le droit positif dans la mesure où elle est appelée à s'adapter aux situations hétérogènes qu'elle rencontre».

6. J.-M. Favret, «Le principe de précaution ou la prise en compte par le droit de l'incertitude scientifique et du risque virtuel», Recueil Dalloz, 2001, p. 3462.

7. Voir A.-M. Casteret, L'affaire du sang, Paris, La Découverte, 1992; M. Massenet, La transmission administrative du sida, Paris, A. Michel, 1992; M.-A. Hermitte, Le sang et le droit: essai sur la transfusion sanguine, Paris, Seuil, 1996; C. Bettati, Responsables et coupables. Une affaire de sang, Paris, Seuil, 1993; S. Paugam, Un sang impur: l'affaire des hémophiles contaminés, Paris, J.-C. Lattès, 1992; E.-L. Henry, De l’hémophilie en général et du crime en particulier, Paris, Le Pré aux Clercs, 1992; L.-A. de Riedmatten, J. Roberto, L'affaire du sang contaminé, Paris, Éditions du Rocher, 1992.

8. Voir L. Lorvellec, «L'action des autorités publiques françaises dans la crise de la vache folle», Revue de droit rural, 1997, p. 219.

9. Voir C. Guettier, «L'amiante: une affaire d'État», Revue de droit sanitaire et social, 2006, p. 202; C. Girault, "Victimes de l'amiante: chronique d'un scandale judiciaire», L'actualité juridique. Droit pénal, 2005, p. 454.

10. M. Setbon, «Le principe de précaution en question", Revue française des affaires sociales, nº 3-4, décembre 1997, p. 201.

11. J.-M. Favret, «Le principe de précaution... », p. 3462; Conseil d’État, Rapport public 1998. Jurisprudence et avis de 1997. Réflexions sur le droit de la santé, Paris, La documentation française (Études et documents du Conseil d'État; 49), 1998: le principe de précaution «interroge notre droit tout entier»; F. Ewald, «La construction du régime juridique du principe de précaution», Recueil Dalloz, 2007, p. 1548.

12. Voir C. Denizeau, «Principe de précaution et droit de l'urbanisme», Revue française de droit administratif, 2012, p. 864. L'article R. 111-15 du Code de l'urbanisme dispose que «le permis ou la décision prise sur la déclaration préalable doit respecter les préoccupations d'environnement définies aux articles L. 110-1 et L. 110-2 du code de l'environnement».

13. C. Lacroix, La réparation des dommages en cas de catastrophes, thèse de doctorat en droit privé et sciences criminelles, université de Haute-Alsace, 2005; éditée par LGDJ (Bibliothèque de droit privé; 490), 2008, nº 1.

14. C. Lienhard, "Pour un droit des catastrophes», Recueil Dalloz, 1995, chron., p. 91.

15. Voir H. Margeat, J.-M. Michel, «La loi sur les catastrophes naturelles», Gazette du Palais, 1984, 2, doctr. nº 500 . Sur la constatation de l'état de catastrophe naturelle, voir CE, 12 mars 1999, Les mutuelles régionales d'assurances, Recueil Lebon, p. 59; CE, 14 mai 2003 , Syndicat des sylviculteurs du sud-ouest, Recueil Lebon, p. 209; L'actualité juridique. Droit administratif, 2003, p. 1449, concl. M. Guyomar. 
Sont considérés comme les effets des catastrophes naturelles $[\ldots]$ les dommages matériels directs ayant eu pour cause déterminante l'intensité anormale d'un agent naturel, lorsque les mesures habituelles à prendre pour prévenir ces dommages n'ont pu empêcher leur survenance ou n'ont pu être prises.

L'affirmation «lorsque les mesures habituelles à prendre pour prévenir ces dommages n'ont pu empêcher leur survenance ou n'ont pu être prises» montre qu'une catastrophe naturelle n'est pas exclusive de la mise en œuvre d'une responsabilité. Dans la société contemporaine, ce lien devient même de plus en plus fort. En effet, les pays les plus développés n'accusent plus la fatalité et développent une attitude active face aux catastrophes naturelles ${ }^{16}$. Leur survenance peut entraîner un questionnement philosophique ${ }^{17}$ mais surtout scientifique sur les causes du malheur. Les derniers exemples de catastrophes naturelles montrent que les scientifiques avancent malheureusement souvent que la catastrophe était évitable ou que ses conséquences auraient pu être réduites! Ces allégations entraînent inévitablement la recherche d'un responsable qui, en raison de son action ou de son inaction, a concouru soit à la catastrophe naturelle en elle-même soit à en aggraver les conséquences ${ }^{18}$.

Dans deux cas récents de catastrophes naturelles - la tempête Xynthia de 2010 et les inondations du Sud de la France de 2015 -, les scientifiques expliquent que l'urbanisation excessive des zones sinistrées a contribué à l'ampleur des dommages. D'abord, concernant les inondations ${ }^{19}$ de 2015, la thèse soutenue est que l'intervention de l'homme, par une urbanisation excessive du Sud de la France, a contribué aux conséquences dramatiques des inondations en empêchant l'écoulement normal des eaux de pluie. M. Wesolek, prévisionniste à l'Observatoire français des orages, explique que «l'urbanisation excessive a empêché les fortes précipitations d'être absorbées par les sols ${ }^{20}$. Il remarque que "cela fait bien longtemps que des experts pointent du doigt l'urbanisation forcenée du Midi qui empêche l'écoulement normal des eaux de pluie ${ }^{21}$. Il en découlerait que:

[...] la nuit apocalyptique vécue par les habitants de la région de Cannes résulte de deux phénomènes d'origine humaine: le réchauffement climatique qui affole la météo et la frénésie immobilière qui a privé le littoral azuréen de ses protections naturelles contre l'eau ${ }^{22}$.

Ensuite, concernant la tempête Xynthia, l'urbanisation excessive du littoral vendéen est aussi mise en cause pour expliquer l'ampleur des dommages de la catastrophe. $\mathrm{M}^{\mathrm{me}}$ Lepage, ancienne ministre de l'Environnement et avocate, explique que:

[...] au-delà des responsabilités locales, ce sont assurément des facteurs clefs de l'excessive «littoralisation» de notre société qui se trouvent, en toile de fond, mis en cause. Il est urgent de stopper, et même de faire régresser l'urbanisation à outrance du littoral, phénomène qui expose à un risque «naturel» avéré la population se concentrant depuis quelques dizaines d'années seulement sur la bande côtière ${ }^{23}$.

Ainsi, il résulterait de l'urbanisation excessive des littoraux pointée du doigt par les experts que «les conséquences tragiques de ces catastrophes naturelles ne doivent rien au hasard $»^{24}$. Il ne s'agirait donc pas «de drames environnementaux, sur lesquels l'être humain n'aurait pas eu de prise ${ }^{25}$. Or, considérer qu'une catastrophe naturelle n'est peut-être pas si naturelle qu'elle en a l'air pose, d'un point de vue juridique, une question de responsabilité ${ }^{26}$. Précisément, en cas de catastrophe naturelle, les élus locaux peuvent-ils, en vertu du principe de précaution, être tenus pour pénalement responsables de ne pas avoir délivré avec suffisamment de parcimonie des permis de construire sur le littoral?

M. le professeur Mayaud remarque que:

Même naturelles, les catastrophes ne sont pas toujours exclues de la main de l'homme, ne serait-ce par les précautions élémentaires qui n'ont pas été prises dans le secteur couvert par l'intervention dommageable des forces qui les ont provoquées: ainsi de constructions dans une zone inondable, de défaut d'interdiction d'un secteur menacé d'éboulement ou d'avalanche, de risques de séismes qui n'ont pas été répercutés à une population ignorante... La nature a ses droits, mais l'homme a des devoirs, qui consistent, sinon à intervenir sur les forces elles-mêmes, du moins à en réduire les conséquences néfastes. C'est ici que le droit pénal intervient, non dans l'impossible, mais dans le possible: autant la responsabilité ne peut être en rapport avec le sinistre dans sa dimension physique et

16. Voir M. Franc, «Traitement juridique...», p. 360

17. Voir J.-P. Dupuis, Pour un catastrophisme éclairé, Paris, Seuil, 2002.

18. Voir D. Viriot-Barrial, «Le droit pénal face aux grandes catastrophes sanitaires», Revue de droit sanitaire et social, 2008, p. 21.

19. Sur les inondations en général, voir D. Canepa, "La sécurité civile face aux inondations ", Les cahiers de la sécurité intérieure, nº 16, 1994, p. 113; P. Dufust, «L'annonce et la prévision des crues. La réorganisation de la transmission des avis des crues », Les cahiers du CFPC, n 17, novembre 1985 , p. 21; F. Duval, "Dommages causés par les inondations", Les annales de la voirie, nº 45, janvier-février 1999, p. 16.

20. Interview diffusée dans le «Journal de $13 \mathrm{~h}$ », France 2, 4 octobre 2015, accessible sur http://www.francetvinfo.fr/meteo/inondations/intemperiesdans-le-sud-est/sud-est-pourquoi-les-intemperies-ont-elles-ete-si-fortes_1112675.html.

21. Ibid.

22. «Inondations en Côte d'Azur: l'urbanisation en question », Le Huffington Post, 5 octobre 2015, en ligne: http://www.huffingtonpost.fr/2015/10/o5/ indondations-cote-d-azur-urbanisation-region_n_8242724.html.

23. «Xynthia: un procès pour que le désastre ne se reproduise pas!», Vendée Nature Environnement, actualités du 18 septembre 2014, en ligne: http:// vne.eklablog.com/environnement-c19064553/3.

24. P. Charrier, «Procès Xynthia: quatre ans de prison pour l'ancien maire de la Faute-sur-Mer», La Croix, 12 décembre 2014.

25. Ibid.

26. Voir B. Fischer, «La loi du 13 juillet 1982 relative à l'indemnisation des victimes de catastrophes naturelles», Revue de droit rural, 1984 , p. 175. 
contraignante, autant elle a légitimement sa place dans ce qui reste abordable pour les décideurs, en termes de prévention, d'information, de sensibilisation ${ }^{27}$.

Ce questionnement relance de manière inédite un débat que l'on pensait $\operatorname{tari}^{28}$ : le principe de précaution doitil pouvoir se frayer une place dans la responsabilité pénale? Cette interrogation est d'autant plus intéressante que dans le cas de la tempête Xynthia, qui a causé la mort de dix hommes, seize femmes et trois enfants, la responsabilité pénale de l'ancien maire de la commune a été engagée. En trois cent seize pages d'un jugement hors norme et à la précision remarquable ${ }^{29}$, le tribunal correctionnel des Sables-d'Olonne a, pour la première fois en France, condamné des élus locaux à une peine de prison ferme pour homicide involontaire pour «n'avoir pas pris les mesures de prévention qui auraient pu empêcher les pertes de vies humaines provoquées par la tempête $»^{30}$. Plusieurs fautes caractérisées personnelles du maire ont été recensées $^{31}$. Il lui a notamment été reproché la délivrance de permis de construire de maisons de plain-pied alors que le projet de plan de prévention du risque inondation (rendu opposable par le préfet) avait imposé un étage, afin d'offrir aux occupants un refuge en cas de tempête. Le jugement fait état d'un risque intentionnellement dénié par l'édile dans un paragraphe intitulé «La connaissance du risque et son déni ». Ce jugement rappelle que, pour réduire l'ampleur des conséquences d'une catastrophe naturelle, la maîtrise de l'urbanisation est essentielle, en particulier lors la délivrance des autorisations d'urbanisme. Cependant, ce n'est pas tant de précaution mais plutôt de prudence que l'édile a manqué puisque le tribunal insiste sur sa connaissance avérée du risque naturel. En effet, le maire

[...] a été destinataire d'une somme considérable d'informations relatives à la connaissance du risque de submersion marine susceptible d'affecter un jour le territoire de sa commune, notamment dans la partie urbanisée située non loin de la digue Est. La communication de ces informations s'est étalée sur une période de douze années, essentiellement en provenance des services de l'État ${ }^{32}$.

Le tribunal insiste sur

[...] une forme de légèreté intellectuelle, consistant à décréter d'une façon irréfragable que la commune de La Faute-sur-Mer demeurerait toujours à l'abri d'une invasion de l'océan, pourtant omniprésent à l'Ouest, au Sud et à l'Est de son territoire et malgré ses particularités géophysiques incontournables ${ }^{33}$.
Enfin, le tribunal explique que:

[...] le prévenu n'a pas plus reconnu le risque comme réel pour l'avenir que pour le passé, et ne l'a jamais personnellement accepté comme crédible, ce qui concourt à expliquer son inertie durant près de 10 ans $^{34}$.

Ce jugement, s'il constitue un outil utile à notre démonstration, ne doit pas pour autant donner lieu à une mauvaise interprétation. En raison des fautes multiples du prévenu et de sa connaissance avérée du risque, il interdit d'en conclure à une quelconque influence du principe de précaution sur la responsabilité pénale de l'élu puisque la principale caractéristique de ce principe, l'incertitude scientifique, fait ici défaut. En outre, l'incertitude scientifique constitue un obstacle infranchissable par la responsabilité pénale. La faute de précaution ne peut donc pas constituer le fait générateur de la responsabilité pénale. De facto, la rencontre entre la responsabilité pénale et le principe de précaution est, en principe, impossible (I). Mais ce jugement montre aussi que la précaution et l'imprudence ne sont pas totalement étrangères l'une à l'autre. Si la première ne doit pas avoir sa place dans la responsabilité pénale, la seconde peut, en revanche - et ce jugement en est une parfaite illustration - entraîner la responsabilité pénale d'un élu local en cas de catastrophe naturelle. Il en résulte que le maintien d'une ligne de démarcation franche entre la prudence et la précaution est indispensable. En effet, l'inverse permettrait une introduction sournoise du principe de précaution dans la responsabilité pénale. La préservation de la conception néoclassique de la responsabilité pénale impose d'empêcher, par ce canal, toute rencontre entre la responsabilité pénale et la précaution (II).

\section{Principe de précaution et responsabilité pénale: une rencontre en principe impossible}

Bien que la logique de précaution ne soit pas totalement étrangère au droit pénal $(\mathrm{A})$, la responsabilité pénale ne peut pas accueillir le principe de précaution comme fait générateur de sa mise en œuvre. En effet, la responsabilité pénale exige l'existence d'un lien de causalité. Or, cette condition constitue, pour le principe de précaution, un obstacle infranchissable (B).

27. Y. Mayaud, «La responsabilité pénale liée aux catastrophes naturelles: quelle défense pour les décideurs locaux?», Revue Lamy collectivités territoriales, $\mathrm{n}^{\circ} 88$, mars 2013 , p. 79.

28. Voir C. Noiville, «La lente maturation jurisprudentielle du principe de précaution», Recueil Dalloz, 2007, p. 1515.

29. Tribunal correctionnel des Sables-d'Olonne, 12 décembre 2014, $\mathrm{n}^{\circ}$ 877-2014.

30. C. Cans, J.-M. Pontier, T. Touret, «Xynthia, ou l'incurie fautive d'un maire obstiné», L'actualité juridique. Droit administratif, 2015, p. 379.

31. Pour un exposé des fautes, voir F. Calvet, C. Manable, Rapport d'information fait au nom de la délégation aux collectivités territoriales et à la décentralisation sur Xynthia, 5 ans après: pour une véritable culture du risque dans les territoires, $\mathrm{n}^{\circ} 536$, enregistré à la présidence du Sénat le 18 juin 2015, p. 15-37.

32. Tribunal correctionnel des Sables-d'Olonne, 12 décembre 2014, no 877-2014, p. 96.

33. Ibid., p. 104

34. Ibid., p. 105 


\section{A. La logique de précaution déjà présente dans le droit pénal}

Le dommage résultant d'une violation du principe de précaution est reconnaissable en ce qu'il est incertain d'un point de vue scientifique. Comme le propre d'un risque est d'être incertain (sinon il ne s'agirait plus d'un risque), cette expression paraît, au premier abord, redondante. La distinction entre existence du risque et survenance du risque est utile à sa compréhension. Si la survenance d'un risque est toujours matériellement incertaine, en revanche, son existence n'est pas toujours scientifiquement incertaine. Le droit pénal est familier avec ces deux facettes: existence du risque et survenance du risque.

Premièrement, un comportement seulement susceptible de produire un dommage peut engager la responsabilité d'un agent. C'est une situation courante et même nécessaire en droit pénal. Il existe des infractions sans résultat (également appelées «infractions à risque »35), c'est-à-dire dont la consommation n'est pas subordonnée à la survenance du dommage. Au sein de ces infractions, la doctrine distingue les infractions-obstacle et les infractions formelles ${ }^{36}$. Ces infractions sont reconnaissables car les textes d'incrimination comportent des expressions comme «de nature à», «dans le but de », «en vue de», ou encore «susceptible de ». L'exemple topique d'infraction à risque est le délit de risques causés à autrui prévu à l'article 223-1 du Code pénal ${ }^{37}$. Le principe de précaution repose donc sur une logique de raisonnement déjà présente en droit pénal.

Deuxièmement, le caractère scientifiquement incertain du risque n'est pas toujours ipso facto un obstacle insurmontable à l'engagement de la responsabilité pénale d'un agent. Les exemples de l'empoisonnement ${ }^{38}$ et de l'administration de substances nuisibles ${ }^{39}$ permettent de s'en convaincre. À première vue, la comparaison entre une infraction intentionnelle et un comportement non intentionnel peut sembler asymétrique. Toutefois, il n'en est rien puisque l'intention est étrangère à notre questionnement, concentré sur le risque comme fait générateur de la responsabilité pénale. Dans l'empoisonnement, le risque visé étant le risque de mort, la substance administrée doit nécessairement être de nature à entraîner la mort ${ }^{40}$. Deux hypothèses doivent être distinguées. Primo, une substance peut être mortelle à très faible dose. Dès lors, en fonction de la dose de produit utilisée, le risque de mort ne présentera aucune incertitude scientifique quant à son existence. Secundo, une substance peut n'être mortelle qu'à certaines doses, à partir d'un seuil pouvant susciter des controverses scientifiques. Dans ce cas, en fonction de la dose administrée, le risque de mort peut devenir scientifiquement incertain. On pourrait penser que la certitude ou l'incertitude scientifique du risque est étrangère à la répression de l'empoisonnement puisque la substance administrée doit seulement être «de nature à» entraîner la mort. Pourtant, le Code pénal prévoit l'incrimination d'administration de substances nuisibles ayant porté atteinte à l'intégrité physique ou psychique d'autrui. Il en découle qu'en cas d'incertitude scientifique du risque de mort, le juge est invité à basculer vers cette qualification. En cela, l'incertitude scientifique fait déjà partie de la logique de raisonnement du juge pénal. Ce critère est déjà indirectement utilisé par le juge dans la qualification des faits sans que personne ne s'en offusque. Dès lors, on pourrait penser qu'il n'y aurait qu'un pas à franchir pour que ce critère devienne opérant en matière d'infraction non intentionnelle.

On pourrait ensuite songer à brandir l'exigence de légalité d'où découle, pour le législateur, l'obligation d'édicter des textes clairs et précis, comme un rempart contre l'incursion du principe de précaution dans la responsabilité pénale. En effet, il parait impossible d'enfermer dans un texte d'incrimination précis la logique de précaution. Celleci repose sur deux composantes trop volatiles: l'existence d'un risque qui, sans être certaine, est à tout le moins plausible en l'état des connaissances scientifiques et le fait que l'agent décisionnaire ne devait pas prendre le risque que le risque arrive. À partir de quel instant un risque devient-il plausible en l'état des connaissances scientifiques? Comment l'agent décisionnaire peut-il distinguer les délivrances d'autorisations d'urbanisme légitimes et sans risque de celles qui font naître un risque incertain? Il semble difficile d'exprimer cela dans un texte suffisamment précis et prévisible. Bien que l'argument semble opérant d'un point de vue théorique, il est plus contestable d'un point de vue pratique. En effet,

Il faut constater, tout en le regrettant, que l'exigence de légalité est assez mal respectée en droit pénal. Il est fréquent que le comportement incriminé ne soit pas défini et, lorsqu'une définition est donnée, il est fréquent qu'elle emploie des standards qui renvoient à l'interprétation du juge et rendent ses limites incertaines ${ }^{41}$.

35. Expression empruntée à D. Roets, «Réflexions sur les possibles implications du principe de précaution en droit pénal de l’imprudence», Revue de sciences criminelles, 2007 , p. 251

36. Voir S. Keyman, «Le résultat pénal», Revue de sciences criminelles, 1968, p. 781; J.-Y. Maréchal, Essai sur le résultat dans la théorie de l’infraction pénale, Paris, L'Harmattan, 2003, p. 334 sq.

37. Art. 223-1 du Code pénal: «Le fait d'exposer directement autrui à un risque immédiat de mort ou de blessures de nature à entraîner une mutilation ou une infirmité permanente par la violation manifestement délibérée d'une obligation particulière de sécurité ou de prudence imposée par la loi ou le règlement est puni d'un an d'emprisonnement et de 15000 euros d'amende»; voir J. Chacornac, "Le risque comme résultat dans les infractions de mise en danger: les limites de la distinction des infractions matérielles et formelles", Revue de sciences criminelles, 2008, p. 849.

38. Art. 221-5 du Code pénal: «Le fait d'attenter à la vie d'autrui par l'emploi ou l'administration de substances de nature à entraîner la mort constitue un empoisonnement».

39. Art. 222-15 du Code pénal: «L'administration de substances nuisibles ayant porté atteinte à l'intégrité physique ou psychique d'autrui est punie des peines mentionnées aux articles 222-7 à 222-14-1 suivant les distinctions prévues par ces articles».

40. Voir D. Mayer, «La notion de substance mortelle en matière d'empoisonnement», Recueil Dalloz, 1994, chron., p. 325.

41. E. Dreyer, «Droit pénal et principe de précaution», Recueil Dalloz, 2015, p. 1912 
Agiter le principe de légalité comme un obstacle à l'immixtion du principe de précaution dans la responsabilité pénale est donc d'une efficacité plutôt réduite.

À ce stade de la réflexion, la logique du principe de précaution semble pouvoir être soluble dans la responsabilité pénale. La rencontre entre les règles d'urbanisme, la responsabilité pénale et le principe de précaution en cas de risque incertain de catastrophe naturelle paraît envisageable. Pourtant, pour être féconde, cette rencontre doit aussi se matérialiser sur le plan du lien de causalité. Or, sur ce point, la rencontre entre les protagonistes ne peut qu'être une rencontre sans lendemain!

\section{B. L'obstacle infranchissable du lien de causalité}

L'exigence de la causalité est incontournable pour pouvoir engager une responsabilité, que celle-ci soit pénale, civile $^{42}$ ou administrative ${ }^{43}$. Dans l'hypothèse qui nous occupe, y a-t-il un lien de causalité entre la délivrance d'autorisations d'urbanisme par l'élu local dans une zone déjà fortement urbanisée et les dommages résultant d'une catastrophe naturelle subséquente? La jurisprudence estime que la causalité ne peut être présumée. Elle a déjà eu l'occasion de le rappeler à propos du délit de risques causés à autrui ${ }^{44}$, délit que certains considèrent d'ailleurs comme une prise en compte du principe de précaution ${ }^{45}$. Ce délit n'est constitué que si le manquement a été la cause directe et immédiate du risque de mort ou de blessures de nature à créer une mutilation ou une infirmité permanente auquel a été exposé autrui ${ }^{46}$. Or, dans le principe de précaution, le caractère scientifiquement incertain du risque a pour conséquence de rendre la causalité ellemême incertaine. M. Gallois considère que:

Si un risque est scientifiquement incertain dans son existence, il est peu probable que le juge fasse fi de cette «réalité» scientifique. Il pourra en tirer les conséquences en considérant que l'existence du risque redouté par l'infraction n'est pas seulement incertaine scientifiquement mais aussi juridiquement. Par conséquent, la causalité juridique reliant le comportement au résultat qui l'a généré sera elle aussi incertaine ${ }^{47}$.
M. le professeur Dreyer considère même que:

L'injustice suprême serait de demander à quelqu'un de répondre pénalement d'un fait dont on n'est pas sûr qu'il se produise ou, s'il s'est produit, dont on n'est pas sûr qu'il trouve son origine dans le comportement de l'agent ${ }^{48}$.

La Cour de cassation a confirmé cette position dans l'affaire du sang contaminé, lorsque le risque de mort inhérent à l'utilisation de poches de sang infectées n'était pas encore évalué avec certitude ${ }^{49}$. Elle a ensuite réaffirmé ce point de vue dans le premier des trois arrêts dits «de l'amiante $»^{50}$. Ce faisant, la haute juridiction refuse d'assimiler la faute de précaution à la faute de prévention. En d'autres termes, elle n'assimile pas l'acceptation d'un risque avéré à celle d'un risque potentiel.

En matière pénale, l'appréciation de la certitude de la causalité précède toujours celle du caractère direct et immédiat. Dès lors, étant incertaine, elle ne saurait être directe et immédiate. Il en résulte que l'incertitude scientifique du risque sanctionné par le principe de précaution empêche irrémédiablement tout engagement de responsabilité pénale. Le choix opéré par le juge pénal entre les qualifications d'empoisonnement et d'administration de substances nuisibles permet à nouveau de justifier cette conclusion. En effet, l'incertitude scientifique est un critère de choix mis en place par le législateur pour permettre au juge de choisir la qualification pénale adéquate. Si le juge pénal se retranche vers la qualification d'administration de substances nuisibles en raison d'une incertitude scientifique sur la nature mortifère des substances, c'est précisément pour que le lien de causalité soit certain (et ensuite direct et immédiat) et que la responsabilité pénale puisse être engagée. En d'autres termes, l'incertitude scientifique pesant sur le caractère mortifère des substances est à l'origine du délaissement de la qualification d'empoisonnement. A contrario, comme la communauté scientifique s'accorde sur le caractère au moins nuisible des substances, l'incertitude scientifique peut être surmontée. Dès lors, le lien de causalité peut exister (et être ensuite direct et immédiat) et la responsabilité pénale peut être engagée. En revanche, en matière de précaution, l'incertitude scientifique est une condition essentielle de l'application du principe. Elle ne pourra donc jamais être surmontée. S'il y a certitude scientifique, ce n'est plus le

42. Voir P. Jourdain, «Principe de précaution et causalité : quelle incidence du premier sur la seconde?», Revue trimestrielle de droit civil, 2011, p. 540 D. Mazeaud, «Responsabilité civile et précaution», Responsabilité civile et assurances, juin 2001, chron. $\mathrm{n}^{\circ} 14$.

43. Voir D. Bailleul, «Évolution de l'appréciation du lien direct de causalité en matière d'urbanisme», L'actualité juridique. Droit administratif 2015, p. 1604; B. Defoort, «Incertitude scientifique et causalité: la preuve par présomption », Revue française de droit administratif, 2008 , p. 549.

44. Cass. crim., 16 décembre 2015, nº 15-80.916: Dalloz actualité, 2 février 2016, obs. D. Goetz; Cass. crim., 12 janvier 2016, n 14-86.503: Dalloz actualité, 28 janvier 2016, obs. D. Goetz.

45. Voir J.-C. Saint-Pau, «La sanction pénale du principe de précaution par le délit de risques causés à autrui», in Des liens et des droits: mélanges en l'honneur de Jean-Pierre Laborde, Paris, Dalloz, 2015, p. 247.

46. Cass. crim., 16 février 1999, nº 97-86.290, Bulletin criminel, $\mathrm{n}^{\circ}$ 24, pour un commentaire, voir Recueil Dalloz, 200o, jurispr., p. 9, note A. Cerf ou encore Revue de sciences criminelles, 1999, p. 581, note Y. Mayaud; Cass. crim., 4 octobre 2005, $\mathrm{n}^{\circ}$ 04-87.654, Bulletin criminel, $\mathrm{n}^{\circ} 250$, pour un commentaire, voir Recueil Dalloz, 2006, p. 1649, obs. G. Roujou de Boubée et Revue de sciences criminelles, 2006, p. 68, obs. Y. Mayaud; Cass. crim., 6 octobre 2009, $\mathrm{n}^{\circ}$ 09-81.037.

47. A. Gallois, «Quelle place pour le principe de précaution dans le droit de la responsabilité pénale?», Revue de droit sanitaire et social, 2013 , p. 801.

48. E. Dreyer, «Droit pénal... », p. 1912.

49. Cass. crim., 18 juin 2003, nº 02-85.199, Bulletin criminel, n 127, pour un commentaire, voir Recueil Dalloz, 2004, p. 1620, note D. Rebut; Recueil Dalloz, 2004, p. 2751, obs. S. Mirabail et Recueil Dalloz, 2005, p. 195, note A. Prothais.

50. Cass. crim., 14 avril 2015, $\mathrm{n}^{\circ}$ 14-85.335: Recueil Dalloz, 2015, p. 868; La semaine juridique, éd. G, 2015, p. 696, note H. Matsopoulou; Gazette du Palais, no 155, 3-4 juin 2015, p. 19, note R. Mesa. 
principe de précaution qui doit être mobilisé. Il en résulte qu'en raison de l'exigence incontournable d'une incertitude scientifique, le principe de précaution ne peut pas, sauf à ruiner la théorie de la causalité, être un fait générateur de la responsabilité pénale d'un agent décisionnaire.

Nous avons souligné, dès les premières lignes de cette étude, la difficulté de définition dont souffre le principe de précaution. Ce flou conduit certains auteurs à considérer que "précaution et prévention sont deux facettes de la prudence ${ }^{51}$, voire que la précaution est «fille de la prudence $»^{52}$. M. le professeur Favret souligne que «la précaution peut être distinguée (ou rapprochée) de la prévention et de la prudence ${ }^{53}$. Cette filiation entre précaution et prudence induit l'idée de l'existence d'une différence de degré, et non de nature, entre le comportement de celui qui passe outre un risque avéré et le comportement de celui qui passe outre un risque éventuel ${ }^{54}$. Ce rapprochement doit être combattu. En effet, il risquerait de permettre la rencontre entre le principe de précaution et la responsabilité pénale. Or, cette rencontre est à empêcher.

\section{Principe de précaution et responsabilité pénale : une rencontre à empêcher}

Comme le notent $\mathrm{M}$. Kourilsky et $\mathrm{M}^{\mathrm{me}}$ Viney en conclusion de leur rapport, le principe de précaution est un outil dont les juges

[...] peuvent [...] faire la meilleure ou la pire des choses: la meilleure, s'ils parviennent à mettre en place des mesures améliorant réellement la sécurité des citoyens, tout en évitant l'écueil d'une démission générale devant toute prise de risque; la pire s'ils le transforment en un carcan excluant toute souplesse et décourageant les initiatives nécessaires à l'innovation et au progrès ${ }^{55}$.

Pour ne pas permettre au principe de précaution de devenir «la pire des choses», il est impérieux de préserver précieusement la distinction entre la précaution et l'imprudence (A). Il y va de la préservation du rôle de la responsabilité pénale (B).

\section{A. Une distinction à préserver entre le principe de précaution et l'imprudence}

La précaution concerne les risques encore hypothétiques ou potentiels. L'imprudence sanctionne les prises de risques avérées. Contrairement à l'absence de précaution, l'imprudence, comme le rappelle la condamnation de l'élu local dans le cadre de la tempête Xynthia, peut engager la responsabilité pénale d'un agent décisionnaire. Les lois du 13 mai $1996^{56}$ et du 10 juillet $2000^{57}$ ont successivement établi un régime de responsabilité tenant compte des spécificités des fonctions des élus. Ainsi, l'article 121-3 du Code pénal disposait d'abord:

Il n'y a point de crime ou de délit sans intention de le commettre. Toutefois, lorsque la loi le prévoit, il y a délit en cas de mise en danger délibérée de la personne d'autrui. Il y a également délit, lorsque la loi le prévoit, en cas d'imprudence, de négligence ou de manquement à une obligation de prudence ou de sécurité, prévue par la loi ou les règlements, sauf si l'auteur des faits a accompli les diligences normales, compte tenu, le cas échéant, de ses missions ou de ses fonctions, de ses compétences, ainsi que du pouvoir et des moyens dont il disposait.

Avec la loi du 10 juillet 2000, l'article 121-3 du Code pénal précise désormais:

[...] Il y a également délit, lorsque la loi le prévoit, en cas de faute d'imprudence, de négligence ou de manquement à une obligation de prudence ou de sécurité prévue par la loi ou le règlement, s'il est établi que l'auteur des faits n'a pas accompli les diligences normales compte tenu, le cas échéant, de la nature de ses missions ou de ses fonctions, de ses compétences ainsi que du pouvoir et des moyens dont il disposait.

Dans le cas prévu par l'alinéa qui précède, les personnes physiques qui n'ont pas causé directement le dommage, mais qui ont créé ou contribué à créer la situation qui a permis la réalisation du dommage ou qui n'ont pas pris les mesures permettant de l'éviter, sont responsables pénalement s'il est établi qu'elles ont, soit violé de façon manifestement délibérée une obligation particulière de prudence ou de sécurité prévue par la loi ou le règlement, soit commis une faute caractérisée et qui exposait autrui à un risque d'une particulière gravité qu'elles ne pouvaient ignorer.

L'intérêt de ce dispositif est de prendre en compte les conditions dans lesquelles le décideur public exerce sa mission tout en permettant de «sanctionner un comportement sans attendre que celui-ci ait tourné à la catastrophe ${ }^{58}$. L'imprudence peut être définie comme le comportement d'un agent qui expose autrui à un risque en manifestant une «indifférence coupable sur le plan social $»{ }^{59}$. La doctrine considère que le législateur opère une distinction entre les imprudences conscientes et les imprudences inconscientes ${ }^{60}$, distinguo qui permet au juge de choisir entre deux types de fautes non intentionnelles. Il en résulterait

51. P. Kourilsky, G. Viney, Le principe de précaution..., résumé introductif.

52. D. Roets, «Réflexions sur les possibles implications...», p. 251.

53. J.-M. Favret, "Le principe de précaution...», p. 3462.

54. E. Dreyer, «Droit pénal...», p. 1912.

55. P. Kourilsky, G. Viney, Le principe de précaution..., p. 214.

56. Loi n 96-393 du 13 mai 1996 relative à la responsabilité pénale pour des faits d'imprudence ou de négligence.

57. Loi no 2000-647 du 10 juillet 2000 tendant à préciser la définition des délits non intentionnels.

58. M. Puech, «De la mise en danger d'autrui », Recueil Dalloz, 1994, chron., p. 153.

59. J. Cedras, «Le dol éventuel: aux limites de l'intention », Recueil Dalloz, 1995, chron., p. 18.

60. S. Corioland, «Responsabilité pénale des personnes publiques (infractions non intentionnelles)», Répertoire de la responsabilité de la puissance publique, 2015, n 125 sq. 
donc que le risque peut être tant avéré que simplement potentiel, les notions de "mise en danger délibérée», $\mathrm{d}^{\prime}$ " imprudence», de "négligence» et de «manquement à une obligation de prudence ou de sécurité prévue par la loi ou le règlement », visées aux alinéas 2 et 3 de l'article 121-3 du Code pénal, n'étant pas exclusives de celle de risque incertain.

Or, le risque incertain est la caractéristique principale du principe de précaution. Si l'imprudence empiète sur son territoire en permettant, elle aussi, de sanctionner une prise de risque incertain, comment différencier un comportement qui relève de l'imprudence d'une attitude dont la précaution fait défaut? Il y a ici manifestement une passerelle dangereuse, pour ne pas dire risquée, entre la précaution et la prudence. M. le professeur Roets résume ainsi la situation:

Il apparaît ainsi que du défaut de prévention (face à un risque avéré) au défaut de précaution (face à un risque potentiel), il n'y a qu'un pas que la seule logique invite à franchir. Il n'est de toute façon guère convaincant d'opposer abruptement "prévention » et "précaution", «risque certain» et «risque incertain», comme en témoignent, par exemple, l'affaire du sang contaminé ou celle dite « de la vache folle». Il est en effet possible d'identifier dans le déroulement de ces deux affaires un no man's land chronologique durant lequel le risque potentiel se transmua progressivement en risque avéré. Aussi, si aucune distinction opératoire ne peut être faite entre prévention et précaution [...] il n'est aucune raison de rejeter a priori l'hypothèse de la responsabilité pénale pour défaut de précaution ${ }^{61}$.

En matière d'autorisations d'urbanisme, la jurisprudence a déjà eu de nombreuses occasions d'engager la responsabilité pénale des élus locaux. Ainsi, la responsabilité pénale d'un élu peut être engagée s'il délivre une autorisation d'urbanisme alors même qu'il sait que cette autorisation entre en contradiction avec les règles d'utilisation des sols ${ }^{62}$. En cas de catastrophe naturelle, la jurisprudence Xynthia illustre le propos. A aussi déjà été engagée la responsabilité pénale d'un élu en cas d'avalanche, si ce risque est particulièrement connu ${ }^{63}$. Dans les hypothèses mentionnées, le risque certain de catastrophe était connu de l'élu qui, imprudemment, a délivré des autorisations d'urbanisme. Ces situations sont distinctes de celles dans lesquelles l'élu délivre des autorisations d'urbanisme alors même que l'existence du risque de catastrophe naturelle n'est pas certaine.

Jusqu'à présent, la question de l'influence du principe de précaution sur le droit de l'urbanisme s'est surtout posée devant le juge administratif, dans le contentieux de l'implantation d'antennes relais ${ }^{64}$. En la matière, «son invocation n'a jamais pu empêcher l'implantation d'un pylône, alors même que les risques sont incertains en l'état des connaissances scientifiques ${ }^{65}$. Devant le juge judiciaire, il nous semble que l'interprétation stricte de l'article 121-3 du Code pénal ne devrait pas permettre de mobiliser la responsabilité pénale en cas de risque incertain ou en amont de la transmutation du risque potentiel en risque avéré. Cette mobilisation prématurée de la responsabilité pénale serait d'abord redoutable du point de vue du lien de causalité. Ensuite, si la responsabilité pénale peut être au service du risque incertain, quelle serait l'utilité du principe de précaution qui deviendrait, en définitive, un doublon de l'imprudence prévue à l'article 121-3 du Code pénal ? Enfin, l'intégration du risque incertain dans le champ de la responsabilité pénale permettrait d'accroître considérablement la répression et pourrait paralyser toute prise de décision des élus locaux. Ainsi, la passerelle que les sophistications de l'article 121-3 du Code pénal paraissent créer entre précaution et imprudence ne doit pas être franchie. Bien que la prudence et la précaution s'inscrivent toutes deux dans une logique de prévention des risques ${ }^{66}$, leur distinction doit être précieusement préservée. Le maintien de cette zone réservée, dans laquelle la responsabilité pénale ne doit pas pouvoir faire irruption, n'a pas pour conséquence d'autoriser l'élu local à ne pas anticiper en matière d'urbanisme. La loi relative à la solidarité et au renouvellement urbains (dite loi SRU) du 13 décembre $2000^{67}$ qui impose aux collectivités locales de réfléchir à l'avenir en matière de risques le démontre. De plus, reconnaître la faute de précaution, aux côtés de la faute d'imprudence, comme fait générateur de la responsabilité pénale dénaturerait le rôle de la responsabilité pénale.

\section{B. La préservation du rôle de la responsabilité pénale}

L'admission de la faute de précaution comme fait générateur d'une responsabilité pénale rénovée entraînerait une mutation du rôle de la responsabilité pénale. Jusqu'à présent, celle-ci résiste aux doctrines positivistes et s'inscrit dans une conception néoclassique de la responsabilité. En matière d'imprudence, le présupposé de l'engagement de la responsabilité pénale est que le risque soit prouvé. C'est pourquoi, il est inconcevable d'envisager la responsabilité pénale d'un élu qui, en délivrant des permis

61. D. Roets, «Réflexions sur les possibles implications...», p. 251.

62. Cass. crim., 14 juin $2005, \mathrm{n}^{\circ}$ 05-80.916.

63. TGI de Bonneville, 17 juillet 2003, n 654-2003.

64. CE, 19 juillet 2010, Association du quartier «Les Hauts de Choiseul», n 328687: J.-B. Dubrulle, «Le principe de précaution dorénavant intégré au droit de l'urbanisme», L'actualité juridique. Droit administratif, 2010, p. 2114; E. Carpentier, «Le principe constitutionnel de précaution, les antennes-relais et le juge administratif», Constitutions, 2010, p. 611.

65. C. Denizeau, «Principe de précaution...», p. 864.

66. Voir G. J. Martin, «Principe de précaution, prévention des risques et responsabilité: quelle novation, quel avenir?», L'actualité juridique. Droit administratif, 2005, p. 2222.

67. Loi $\mathrm{n}^{\circ}$ 2000-1208 du 13 décembre 2000 relative à la solidarité et au renouvellement urbains. 
de construire dans une zone excessivement urbanisée, pourrait éventuellement exposer autrui ou un bien à un risque incertain de mort ou de destruction consécutif à une catastrophe naturelle. Probatio diabolica! Le requérant ne peut pas prouver l'existence d'un risque certain puisque précisément le risque est incertain. Tout au plus, il pourra prouver l'existence d'une incertitude! Sur ce point, M. le professeur Martin note que:

[...] il convient de veiller à ce que des incriminations telles que celle qui vise à réprimer la mise en danger d'autrui, par exemple, soient interprétées par référence explicite aux éléments qui la constituent (la conscience du risque et de sa gravité, en l'espèce, qui semble exclure l'incertitude) et qu'elles ne voient pas leur domaine abusivement élargi par référence à des principes dont la généralité s'apparente mal à la nécessaire protection des libertés ${ }^{68}$.

Le délit de mise en danger ne devrait donc pas pouvoir permettre de sanctionner l'exposition d'autrui à un risque hypothétique grave d'atteinte à la vie ou à l'intégrité de la personne. Seule une réécriture de l'article 223-1 du Code pénal permettrait d'atteindre cet objectif. Le législateur pourrait aussi imaginer créer des incriminations particulières inspirées du principe de précaution pour engager la responsabilité pénale d'un agent décisionnaire peu précautionneux. Cette piste peu respectueuse de l'orthodoxie juridique, en particulier par rapport au lien de causalité, entraînerait la responsabilité pénale dans un courant «d'exercice optimisé des droits des victimes au cours des procès des catastrophes ${ }^{69}$. Il en résulte que le principe de précaution semble fondamentalement inadapté à la responsabilité pénale.

Au sujet du droit de propriété, $M^{\text {me }}$ le professeur Le Pourhiet remarque que:

Lorsque le philosophe Marcel Gauchet écrit qu' « un nouvel homme des droits de l'homme est né qui n'a plus rien en commun avec son ancêtre de 1789 ", il exprime ce que tout juriste un tant soit peu sociologue et philosophe observe au quotidien, c'est-à-dire une sorte de Canada Dry juridique où cela s'appelle bien toujours des droits de l'homme mais ce ne sont plus les droits de l'homme ${ }^{70}$.

Le droit de l'urbanisme a toujours été un des acteurs de cette mutation. Dans un premier temps, il a permis la désacralisation du droit de propriété au profit d'une socialisation du droit de propriété. Il est aujourd'hui l'acteur d'un basculement de la socialisation vers la sociétalisation du droit de propriété. Le droit de l'urbanisme ne doit pas poursuivre ce mouvement en entraînant, via le principe de précaution, la responsabilité pénale, dans un courant de socialisation, voire de sociétalisation. Sous le masque du progrès, la responsabilité pénale en sortirait appauvrie ${ }^{71}$. Sa finalité doit rester la répression des comportements dangereux ou asociaux. Ce but est atteint en matière d'imprudence. En effet, lorsque le risque est certain (exemple: tempête Xynthia), l'élu a le choix entre ignorer le risque ou agir pour le réduire. Si la catastrophe survient, il pourra lui être reproché d'avoir agi de manière imprudente. La mise en œuvre de sa responsabilité pénale sanctionne le mauvais exercice de sa liberté de choix. La finalité est donc bien de réprimer un comportement dangereux. Au contraire, lorsque le risque est incertain, les choix de l'agent ne peuvent être fautifs ni être considérés comme dangereux puisque le risque en lui-même n'est pas établi avec certitude. La logique de précaution ne peut donc pas coïncider avec la logique de la responsabilité pénale. Celle-ci n'a pas vocation à devenir un instrument de régulation des comportements sociaux. Le principe de précaution n'a donc pas, à nos yeux, un potentiel d'innovation juridique par rapport à la responsabilité pénale qui n'est pas l'outil adéquat pour construire la société de la prévoyance qui était chère à Jacques Ellul ${ }^{72}$.

68. G. J. Martin, «Principe de précaution...», p. 2222.

69. C. Lacroix, La réparation des dommages..., $\mathrm{n}^{\circ} 555$ sq.

70. A.-M. Le Pourhiet, «Le droit de propriété: du sacré au profane», en ligne: http://www.icrei.fr/wp-content/uploads/PDF_FR/LePouhietenfrancais.pdf.

71. Voir G. J. Martin, «Précaution et évolution du droit», Recueil Dalloz, 1995, chron., p. 299. Voir également l'échange entre C. Bébéar, «Non aux ayatollahs de la prudence", Le Monde, 25 octobre 2007, et B. Latour, "Vive l'audacieux principe de précaution", Le Monde, 5 novembre 2007.

72. J. Ellul, Le bluff technologique, Paris, Hachette, 1988, p. 122 ; cité par D. Roets, «Réflexions sur les possibles implications...», p. 251. 